\title{
Quantitative Morphology of Dermal Elastic Fibers System of the Human Face during Aging
}

\author{
By \\ Iwao SATO, Ryuji UENO, Masataka SUNOHARA and Toru SATO \\ Department of Anatomy, School of Dentistry at Tokyo, The Nippon Dental University, \\ 1-9-20 Fujimi, Chiyoda-ku, Tokyo, 102, Japan \\ - Received for Publication, May 7, 1997 -
}

Key Words: Elastic fiber, Human face, Scanning electron microscope

\begin{abstract}
Summary: Human skin has various distributions and arrangements of elastic fiber (EF). Previons reports did not clearly show the distribution of EF in the face skin because of various contents during aging. In this study, a color image analyzer indicated distribution of elastic, oxytalan, and muscle fibers in human face skin. During aging the muscle fiber size and the content of the EF decreased in the modiolus and inferior labial regions of the human skin, and the ratio of the EF was lower than that of oxytalan fiber in measured areas. That is, the dimension of oxytalan fiber may reflect the content of EF, and muscle has a role in the distribution of the EF in human face skin. In the deeper regions, small and large EF bundles were found near the sheath of gland and muscles. Therefore, face movement might be an important aspect to maintaining the EF content of human face skin.
\end{abstract}

While elastic fiber is found in various organs, previous reports have not clearly shown the distribution of EF due to variations; a thickening (Mongtana, 1973), decrease in number and thickness (Mitchell, 1967), and no significance at light microscopic levels (Marshall, 1965), and also specific ultrastructural properties (Stadler et al., 1978; Tsuji and Hamada, 1981) during aging. The majority of previous observations were only obtained from a region beneath the epithelium. Deeper than this, the distribution of elastic fiber has not been examined. Deeper regions contained muscle fibers might change configuration.

Our analysis permits more detailed information in a quantitative distribution of elastic fibers taking into consideration of age-related changes. Therefore, the purpose of this study was to determine the distribution of the elastic fiber in the human face skin from the epidermal area to deeper regions connected to muscle fibers and to relate these findings to morphological properties.

\section{Materials and Methods}

Twenty adult humans were selected autopsy from donations (males, aged 27 to 70 years of ages) for a histochemical analysis at microscopic and scanning electron microscopic levels. The materials were removed rapidly and immediately fixed in $10 \%$ formalin for one day at $4^{\circ} \mathrm{C}$. After being washed in water, they were dehydrated in absolute ethyl alcohol and then embedded in paraffin. Serial frontal sections were cut at a thickness of about $3 \mu \mathrm{m}$ on a rotary microtome. Sections were stained using the following methods: (1) Elasticavan Gieson staining was performed to demonstrate the locations of elastic fibers and (2) Elastica-van Gieson staining after incubation in a preoxidizing solution (pH 1.5), including $0.3 \% \mathrm{KMnO}_{4}$ and $0.3 \%$ $\mathrm{H}_{2} \mathrm{SO}_{4}$, was performed to demonstrate oxytalan fibers (a modified version of the method of Fullmer, 1959). Serial sections were observed using a real color image system (Swallo II, Interquest, Osaka, Japan). This system was configured with a color image measurement system using a Victor video camera with control unit and a Digital VX color video monitor linked to a light microscope (Vanox- 
S; Olympus, Tokyo, Japan). Measurement areas (rate of elastic fiber in $10,000 \mu \mathrm{m}^{2}$, cross-section of muscle fiber), were selected from skin surface to inner region; the papillary dermis, the intercellular substance, and the connective tissues with the muscle fibers among five regions $\left(1 \mathrm{~cm}^{3}\right)$; frontal, orbital, superior labial, modiolus, and inferior labial regions.

For ultrastructural observations, specimens were etched with $8 \mathrm{~N} \mathrm{HCl}$ for 30 minutes at $60^{\circ} \mathrm{C}$ after fixation with $0.2 \mathrm{M}$ glutaraldehyde solution. They were then post-fixed in a $1 \%$ solution of osmic acid, washed with $2 \%$ cacodylate buffer ( $\mathrm{pH} 7.2)$, and finally dried using a t-butyl alcohol freeze-drying method. These specimens were coated with a 1.5-nm layer of gold-palladium and observed under a Hitachi S-4000 scanning electron microscope (SEM).

\section{Results}

Distribution of elastic fiber with Elastica-van Gieson staining

In the specimens, elastic fibers (EFs) were located in the epithelium, intercellular substance, connective tissue near muscle fiber, and the perimysium and endomysium of the muscle fiber. In the thick intercellular substance concentrated EFs were found, and they varied in arrangement in each five of the observed portions (Fig. 1).

The arrangement of the EFs varied in each re- a

\begin{tabular}{|c|c|c|c|}
\hline age & PD & IS & $C N$ near $M L$ \\
\hline $20 N$ & * & $*$ & * \\
\hline 40 & $*$ & $※$ & $*, *$ \\
\hline 50 & $*, *, \|$ & $※, ※$ & $※$ \\
\hline 60 & ※ & $*$ ※ & ※ \\
\hline 70 & ※,* & * & * \\
\hline 80 & * & * & ※ \\
\hline
\end{tabular}

c

\begin{tabular}{|c|c|c|c|}
\hline age & PD & IS & $C N$ near $M L$ \\
\hline $20 N$ & * & * & * \\
\hline 40 & $\begin{array}{llllll}\dagger & & l\end{array}$ & $=※$ & $*$ \# \\
\hline 50 & $\dagger$ & $=$,※,※ & $*$ \\
\hline$\infty$ & $1, \%$ & $\nVdash, ※$ & 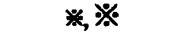 \\
\hline$\pi$ & $1, *$ & $※$ & ※ ※ \\
\hline 8 & * & $*, *$ &,$※$ \\
\hline
\end{tabular}

e

\begin{tabular}{|c|c|c|c|c|}
\hline age & PD & is & $\mathrm{CN}$ near $\mathrm{ML}$ & $M L$ \\
\hline $20 N$ & $\dagger$ & $=$ & $*, *$ & * \\
\hline 40 & 1 & ※ & ※ & $*$ \\
\hline 50 & 1 & ※ & ※ & $*$ \\
\hline 60 & 1 & * ※ & $※$ & $*$ \\
\hline 70 & * & * & $※$ & $*$ \\
\hline 80 & 1 & \#, ※ & ※ & *, \\
\hline
\end{tabular}

\section{b}

$\mathrm{ML}$
$*$
$*$
$*$
$*$
$*$

age
201
40
50
60
70
80

$\begin{array}{ll}\text { PD } & \text { IS } \\ \dagger & * \\ \dagger & \# \\ \|, *,= & \# \\ =, * & * \\ * & *, * \\ * & * \ldots\end{array}$

$\begin{array}{cc}\text { CN near ML } & \text { ML } \\ ※ & * \\ *, * & * \\ * & * \\ ※ & * \\ ※ & * \\ ※ & *\end{array}$

\section{d}

\begin{tabular}{|c|c|c|c|c|}
\hline ML & age & PD & IS & $C N$ near $M L$ \\
\hline$*$ & $20 N$ & $\dagger$ & $=$ & $\nVdash, ※$ \\
\hline$*$ & 40 & 1 & $=$ ж & $*$ * \\
\hline$*$ & 50 & 1 & $=$, & *, \\
\hline$*$ & 60 & $1, *$ & $*, \%$ & *※ \\
\hline$*$ & 70 & $1, *$ & $※, ※$ & $*, \%$ \\
\hline & 80 & $1, *$ & $*$ & $* * *$ \\
\hline
\end{tabular}

Fig. 1. Morphology of elastic fibers from the surface to muscle layer of human face. a: Frontal region; b: Orbital region; c: Superior labial region; d: Modiolus region; e: Inferior labial region. Abbreviation; PD: Papillary Dermis; IS: Intercellular Substance; CN: Connective Tissue; ML: Muscle Layer. Morphology of EFs: Simple (Fine bundle, *; Large bundle, *); Candelabra-like structure (Fine bundle, $\dagger$; Large bundle, $\dagger$ ); Complicated (Fine bundle, $*$; Large bundle, $※$ ); Network (\#) Vertical arranged (Fine bundle, $\|$; Large bundle, $\|$ ); Parallel arranged (Fine bundle, =; Large bundle, =). 
gion. In the frontal region (frontal bell of occipitofrontalis) of the papillary dermis, a large rough EFs ran regularly parallel to the skin surface. In the intercellular substance numerous complicated collagenous fibers containing large and small EFs were found around a hair follicle and a sweat gland. Large and small EFs were found among the collagenous bundles of the connective tissues near the muscle fibers. Only a few EFs were found around the connective tissue of the perimysium and endomysium.

At the orbital region (orbital part of Orbicularis oculi), the EF ran vertical to the surface layer and formed candelabra-like structures in the papilliform connective tissues beneath the epidermis surface layer. Numerous large and small EFs formed networks arranged among collagenous bundles of the intercellular substance. Numerous large EFs were found among the collagenous bundles of the connective tissues near the muscle fibers. A few large EFs were irregularly arranged in the connective tissues of the perimysium and endomysium.

At the superior labial region (Orbicularis oris), small vertical EFs were candelabra-like structures in papilliform connective tissues beneath the epidermis surface layer. They connected with the numerous complicated large EFs which ran parallel to the skin surface. The connective tissues contain numerous large and small EFs forming a complex network among the collagenous bundles of the intercellular substance. Connective tissues containing network-like structures of EF formed layers also found between muscle bundles. A few of EFs were found around the connective tissue near the perimysium and endomysium.

At the modiolus region, small vertical EFs were candelabra-like structures in papilliform connective tissues beneath the epidermis surface layer. They were connected to a complicated arrangement of parallel EFs. The connective tissues contain numerous large and small EF. A few of EFs was found around the connective tissue near the perimysium and endomysium.

In the inferior labial region (Depressor labii inferioris), fine EFs ran vertical to the surface layer showing candelabra-like structures in the papillary dermis. Numerous large EFs running parallel to the surface connected with these fibers. Numerous large and small EFs were found among collagenous bundles of the intercellular substance. Numerous EF forming networks were also found among the muscle fibers.

In the other specimens, from ages 50 to 80 years of age, the EFs were also located in the epithelium, intercellular substance, and connective tissue, however, the distribution in these regions was different.
During aging, the distribution and morphology of EF changed especially at the papillary dermis. Intercellular substance at older ages compared to that of younger ages were as shown in tables (Figs. 1,3).

\section{Dimension of elastic fiber, oxytalan fiber and muscles in human skin}

At the five examined regions, the ratio of elastic fiber (20-80 years of age) and oxytalan (20 years of age) were measured using a real color image system linked to a light microscope. The amount of EF decreased from the superficial portion to inner portions, and this tendency was consistent in each region except for that of the frontal region of the human face. In the modiolus and inferior labial regions, the reduction rate of the EF was high. Comparing the EF and the oxytalan fiber, the ratio of the EF was lower than that of oxytalan in almost every region (Figs. 3, 4). In measurement regions, the cross-sectional area of muscle fiber decreased rapidly, and was especially marked in the modiolus region (Fig. 5).

\section{Scanning electron microscopy}

The connective tissue of the EFs were composed of irregularly arranged small (about $0.1 \mu \mathrm{m}$ in diameter) and large fibers (about $0.5 \mu \mathrm{m}$ in diameter) from the papillary dermis to intercellular substance. A few very fine irregularly arranged fibers forming a network, were found around large EF bundles. The EF composed of large bundles were found around the network of collagen fibers. These large bundles were irregularly connected to the other collagenous complicated fibers in almost all examined regions. In the intercellular substance, branches of the EFs (about $300 \mathrm{~nm}$ in diameter) linked with the collagenous sheath of the sweat gland. Small EFs (about $150 \mathrm{~nm}$ in diameter) with numerous collagenous fine fibers were found among the connective tissue of the perimysium and endomysium (Fig. 6).

\section{Discussion}

Described several aspects of quantitative analysis of the EF based on dissection materials (Mongtana, 1973; Mitchell, 1967; Marshall, 1965). In our study, the methods made histochemical simple staining modified from Fullmer (1959) possible with no nuclear staining in which more differentiation between elastic fiber and collagen fiber system could be determined using a photometric 41,000 color analysis software system. The method using digital analysis of elastic fiber was similar to that of Moloney et al. (1992) reports, however, it is 

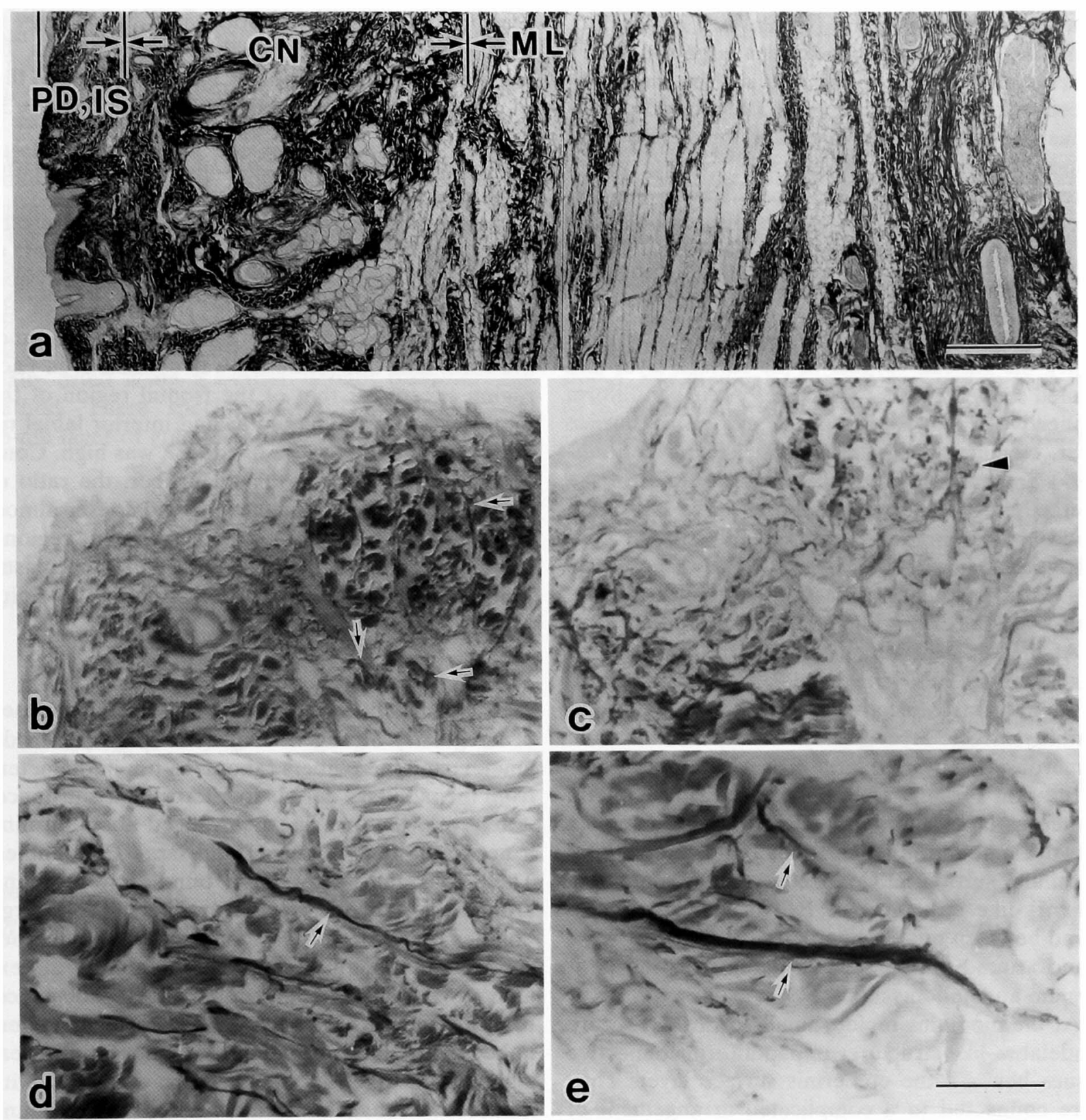

Fig. 2. A light micrograph of the human skin of the sectioned. a) Skin surface to inner region; the papillary dermis, the intercellular substance, and the connective tissues near the muscle fibers. b) In the papillary dermis some of the fibers are seen to run irregularly, forming a complex network with elastica-van Gieson staining. c) In the papillary dermis, a serial section with elastica-van Gieson staining after incubation in a preoxidizing solution to demonstrate oxytalan fibers of fib. 1b. d) In the intercellular substance, the large bundles of fibers are seen with elastica-van Gieson staining. e) In the intercellular substance, a serial section with elastica-van Gieson staining after incubation in a preoxidizing solution to demonstrate oxytalan fibers of fig. 1d. Abbreviation; CN, connective tissue, IS, intercellular substance; ML, muscle layer, PD, papillary dermis; arrow, elastic.fiber; arrow head, oxytalan fiber, bar $=50 \mu \mathrm{m}$.

possible that the analysis does not always coincide with the distribution of elastic fiber because of nodifferentiation analyzing of nucleus in tissues using a chronic ultraviolet irradiation. Our study was based on elastic fiber system, no-staining nuclear methods and a color image analysis. Moreover, the measurement areas from the superficial to deep regions of human skin provide more detailed information about morphological features compared to previous reports.

In our results, the ratio of oxytalan fiber in areas under the measurement was more than that of the elastic fiber. In general, oxytalan fibers are composed of elastic, pre-elastic and other fibers 
a

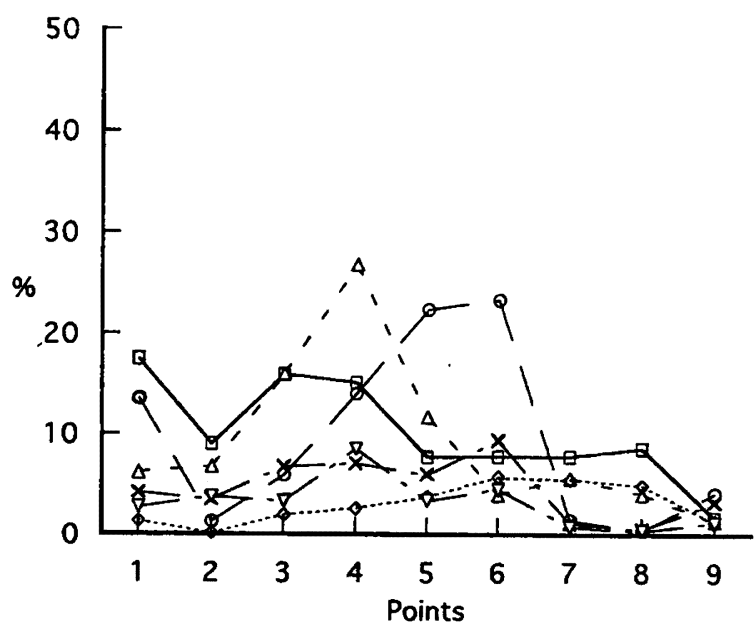

C

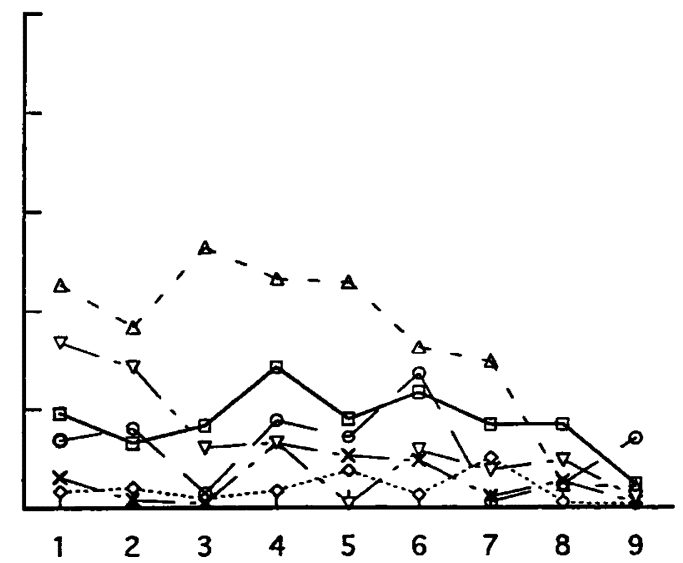

e

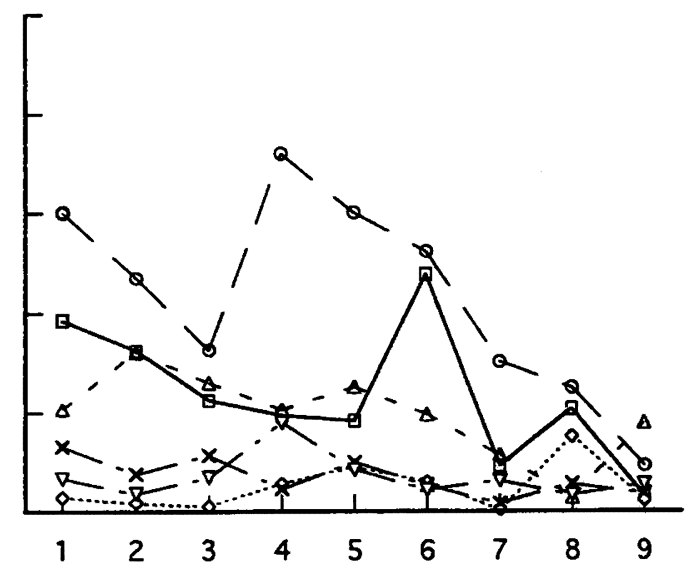

b

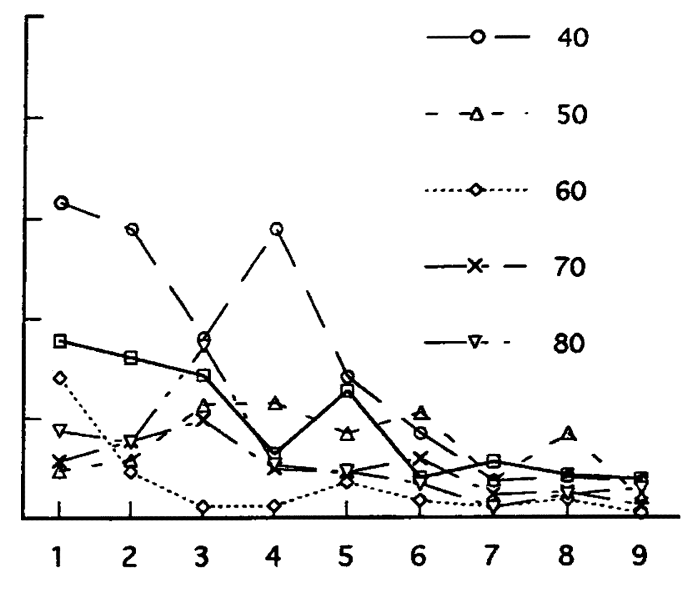

d

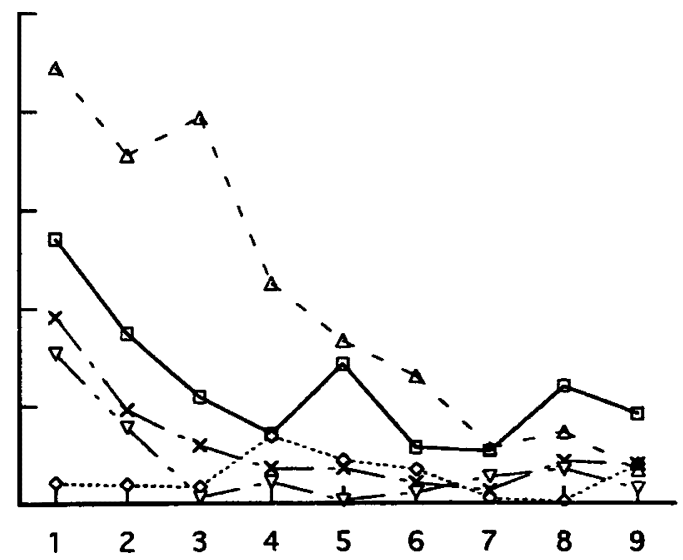

Fig. 3. Distribution of elastic fiber in 20-28 years of age human skin. Measurement areas (ratio of elastic fiber), were selected from skin surface to inner region; points 1-2, the papillary dermis, points 3-5, the intercellular substance, and points 6-9, the connective tissues with the muscle fibers. a, frontal; b, orbital; c, superior labial; d, modiolous; $e$, inferior labial region. 


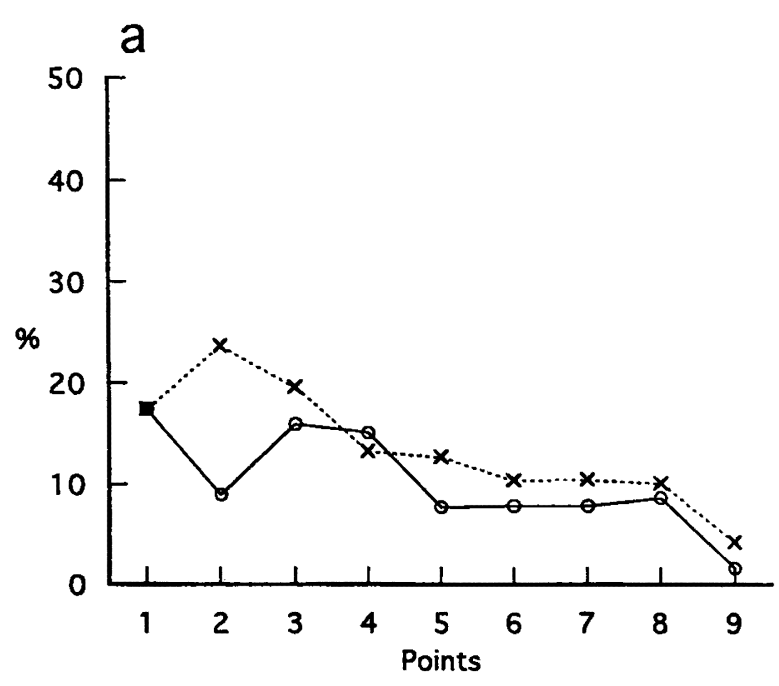

b
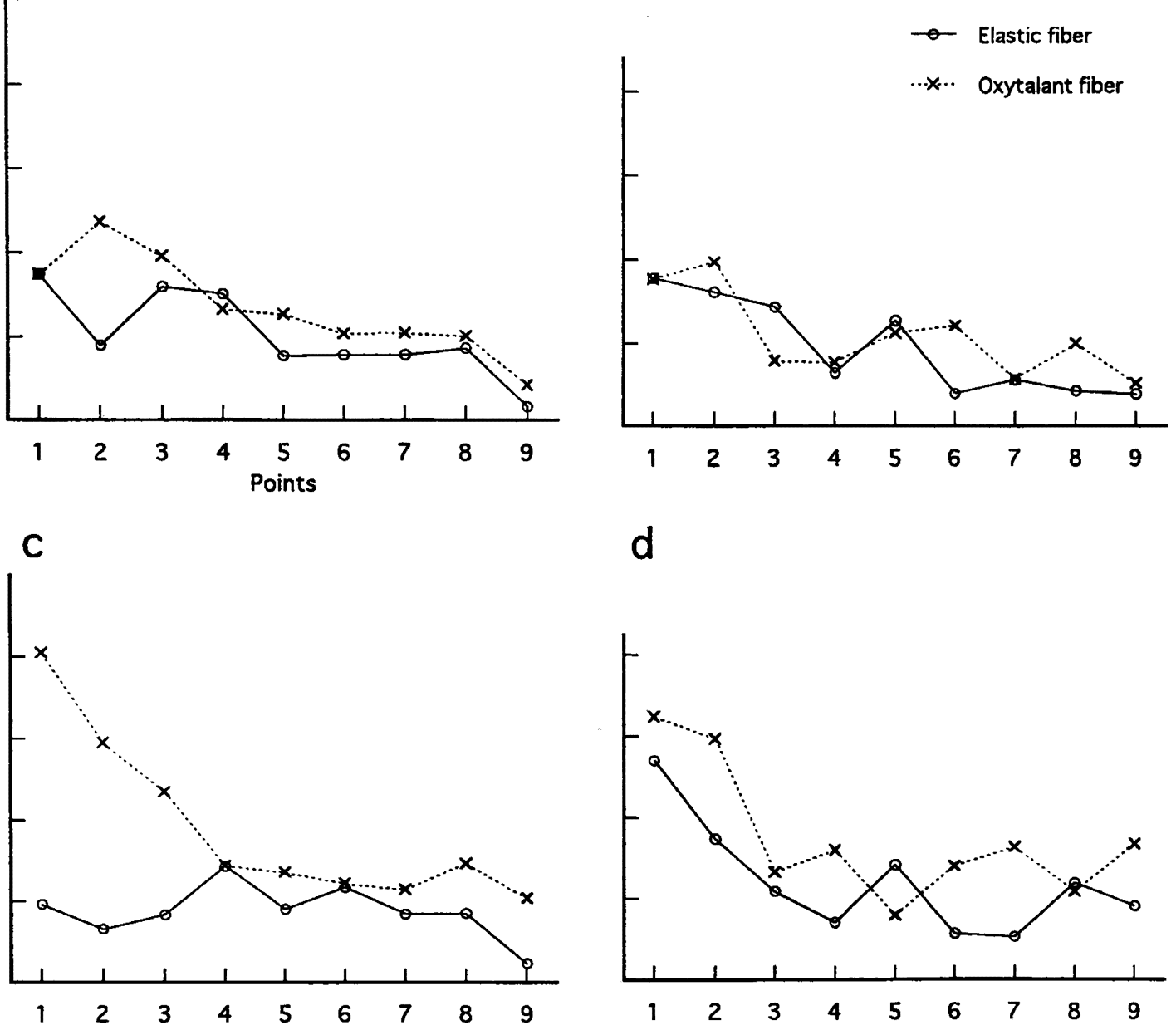

e

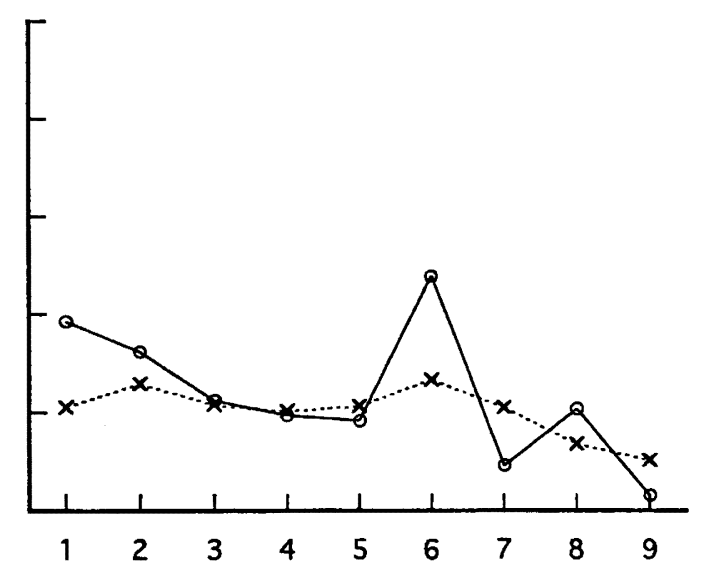

Fig. 4. Distribution of elastic fiber and oxytalant fiber in human skin in 20 years of ages. Measurement areas is same region in Fig. 2. 


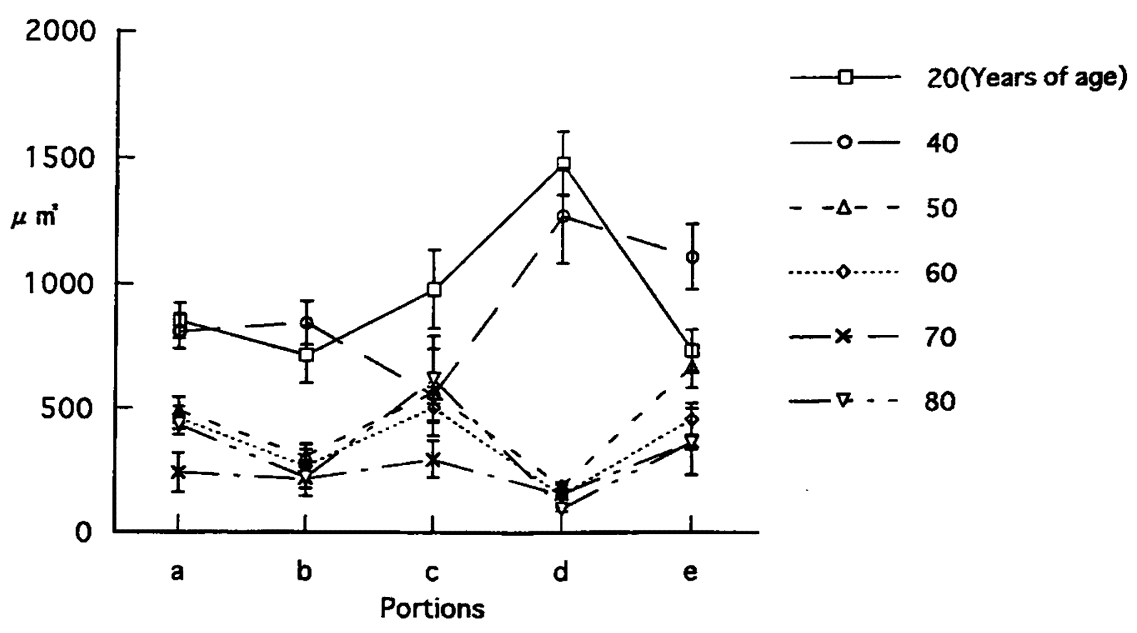

Fig. 5. The cross-sectional area of facial muscle. Abbreviation: a, frontal region; b, orbital region; c, superior labial region; d, modiolus region; e, inferior labial region.

(Fullmer, 1959). If the pre-elastic fiber changes into elastic fiber during aging, this suggests a possible reason for increased quantity of elastic fibers as Montagna report (1973). Therefore, it is able to interpret the increased quantity of elastic fiber in the superior labial and modiolus regions of the 50 yearold in our results, that is, the oxytalan fiber changed into elastic fiber in these regions. The ratio oxytalan fiber was specifically higher than that of the elastic fiber in these two regions. The increase of the elastic fiber may be consequence of the quantity of oxytalan fibers.

The protecting force of this region of the human skin is reflected in the hardening of skin. Reduction of elasticity of human skin was found to occur from 30 years of age (Linda and Noro, 1995) and the EF was consistent to that indicated of the morphology by Tsuji and Hamada (1981). Kligman et al. (1985) reported the change of morphology that occurred in the wrinkles of human skin. In the skin, the morphology and distribution of the EF changed during aging which reflect to functional properties of facial skin. Compared to quantitative elastic fiber in human face skin, the ratio of the EF in sample of 60 years of age rapidly decreased in contrast to that of the 50 years of age. While, our results showed the occurrence of change for morphologies from 60 years of age, they do not however, always allow for age-related changes in face skin.

On the other hand, there is a specific relation between the distribution of elastic fiber and dimension of muscle fibers. If the dimension of muscle fibers changed during aging, the quantitative elastic fiber might also be changed due to the physiological deterioration. It is therefore most plausible to assume that the loss of mass in muscle fibers leads to the decrease of EF, such as is known to occur in facial muscle and skin at old ages. During aging other collagenous mass increased. The occurrence of wrinkles might be connected to collagenous mass change with the frame work of connective tissues in facial skin. A muscle physiological property gave a significance to the ratio of EF in the modiolus portion. In this portion oral muscles participate in sucking and masticatory movements. Until 40 years of age, the size of this muscle fiber is larger than that of others. These differences muscle fiber size and the distribution of EFs were found in the same stages. Aging might effect the muscle property at first, then the elastic fiber, and continue until finally the connective tissues mass is changed. Morphological features were found in the connective tissues of three regions; epithelium, intercellular substance, and connective tissue with muscle fibers, which suggested the specific protecting effect in each observed region. At ultrastructural levels, EF bundles were found near the collagenous sheath of the sweat gland and the connective tissue of the perimysium and endomysium among the muscles. During mouth movement, the EF may act as protection and stretch to these organs and cause the secretion of sweat glands during muscles movements. Compared to the distribution of the EF at a light microscopic level, our results indicated the ratio of the EF was most changed in the modiolus and inferior labial regions, therefore, these regions might effect of physiological stress during aging. 

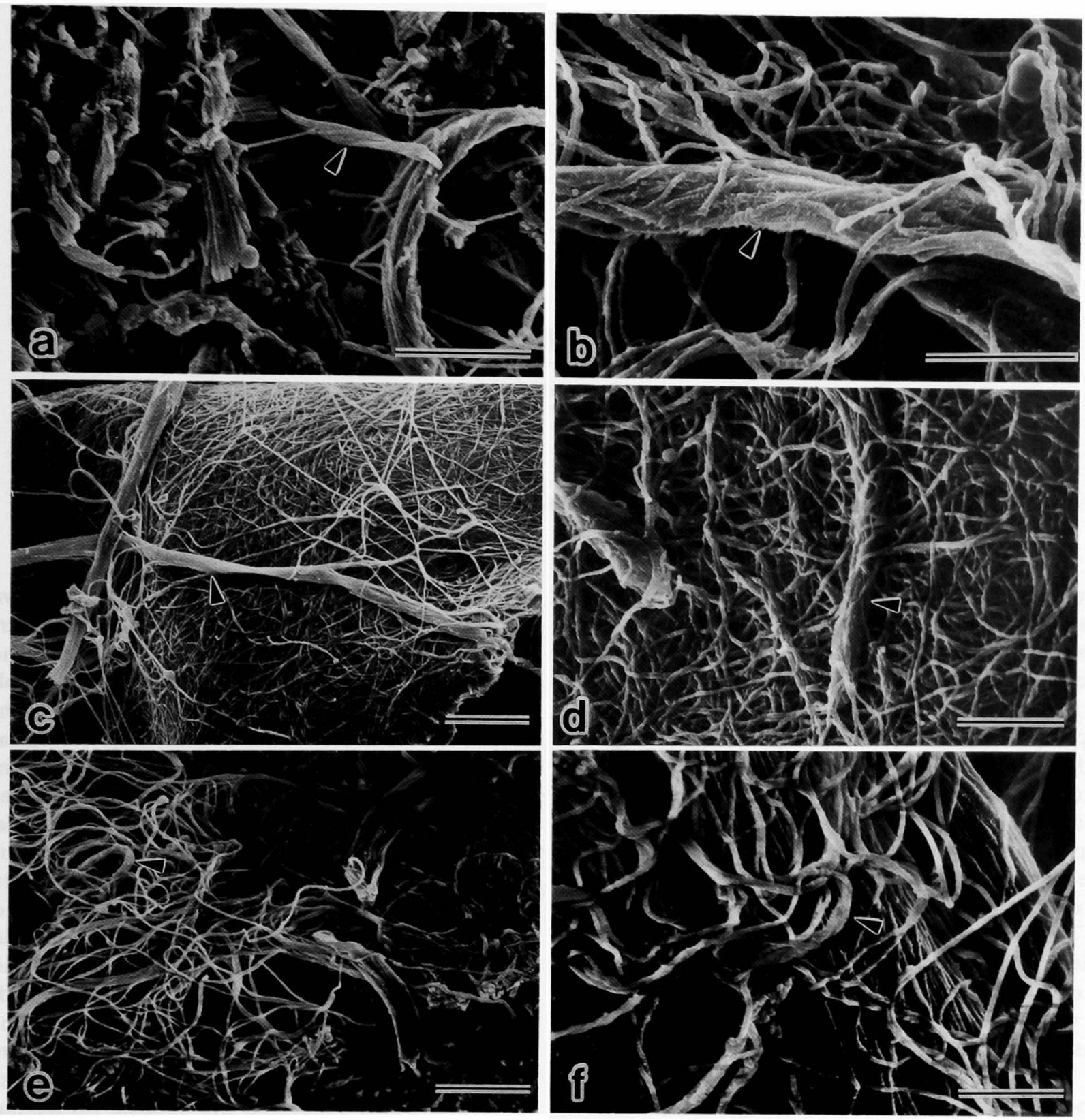

Fig. 6. Scanning electronic microscopy of human skin. a) In the papillary dermis bundle fibers are found in the compound collagenous fibers $(\mathrm{bar}=2 \mu \mathrm{m})$. b) In the intercellular substance, the large bundles of the EF with numerous collagen fibers are composed of some of elastic fibers $(\mathrm{bar}=1 \mu \mathrm{m}) . \mathrm{c}, \mathrm{d})$ In the intercellular substance, branches of the $\mathrm{EF}(\mathrm{c}, \mathrm{bar}=2 \mu \mathrm{m}$; $\mathrm{d}, \mathrm{bar}=1 \mu \mathrm{m}$ ) linked with the sheath of the sweat gland. e, f) Small EF with numerous collagenous fine fibers were found among the connective tissue of the perimysium $(e, b a r=2 \mu \mathrm{m})$ and endomysium $(f, b a r=1 \mu \mathrm{m})$ arrow, elastic fiber. 


\section{References}

1) Fullmer HM. Observation on the development of oxytalan fibers in the periodontium of man. J Dent Res 1959; 38:510-518.

2) Kligman AM, Zheng $P$ and Lavker $R$. The anatomy and pathogenesis of wrinkles. Br J Dermatol 1985; 113:37-42.

3) Lida I and Noro $K$. An analysis of the reduction of elasticity on the aging of human skin and the recovering effect of a facial massage. Ergonomics 1995; 38:1921-1931.

4) Marshall D. The clinical and pathological effects of prolonged solar exposure. I - The association with aging of the skin. Aust. N Z J Surg 1965; 34:161-172.

5) Mitchell RE. Chronic solar dermatosis: A light and electron microscopic study of the dermis. J Invest Dermatol
1967; 48:203-220.

6) Moloney SJ, Edomonds SH, Giddens LD and Learn DB. The hairless mouse model of photoaging evaluation of the relationship between dermal elastin, collagen, skin thickness and wrinkles. Photochem. Photobioi. 1992; 56:505511.

7) Mongtana W. Aging of the nipple and areola. Minerva Dermatol 1973; 108:3-11.

8) Stadler $R$ and Orfanos CE. Reifung und Alterung der elastischen Fasern. Electronmikroskopische Studien in verschiedenen Altersperioden. Arch Dermatol Res 1978; 262:97-111.

9) Tsuji $T$ and Hamada $T$. Age-ralated changes in human dermal elastic fibers. Br J Dermatol 1981; 105:57-63. 